\title{
Preliminary Results of a Technology Demonstration of the Internet Routing in Space Capability over a Geostationary Communications Satellite
}

\author{
Enrique G. Cuevas, Ph.D. \\ Zhuangbo Tang, Ph.D. \\ Applied Physics Laboratory \\ Johns Hopkins University
}

Keywords: IRIS, OBP, GSO, Satellite, VPN, BoD, QoS.

\begin{abstract}
The Internet Protocol Routing in Space (IRIS) was developed by an industry group, under the auspices of the United States Department of Defense ${ }^{1}$ as a Joint Capability Technology Demonstration (JCTD). The primary goal of the IRIS JCTD is to assess the network capability and operational utility of an IP router in space. This paper discusses the results obtained from laboratory and field tests which were specifically designed to evaluate the functionality and performance. Most of the results were obtained during a recent demonstration using 3 ground terminals operating with the IRIS payload on-board INTELSAT-14 (IS-14). Emphasis is given to application and network performance resulting from the end-to-end Quality of Service and Bandwidth on Demand capabilities of IRIS.
\end{abstract}

\section{Introduction}

During the past three years, a significant collaborative effort between industry and government took place with the goal of placing an IP router on-board a commercial geostationary orbit satellite and demonstrating the utility to government users [1]. The IRIS Next Generation Global Services (NGGS) network was developed by an industry team led by Cisco Systems, Inc. The network consists of the IRIS payload on-board the spacecraft; ground based IRIS-compatible terminals and an IRIS Network Operations Center (NOC) [2]. After significant development and testing efforts, the IRIS payload on-board IS14 was launched successfully into orbit on November 23, 2009.

In order to assess this new capability and the potential benefit to government users, the IRIS JCTD was established under the auspices of the United States Department of Defense. The assessment approach consisted of a series of demonstrations leading to a final on-orbit demonstration and assessment, upon the launch of the spacecraft. Operational Demonstrations (OD) with user group participation were designed to assess the operational utility of IRIS, while laboratory and field

1 This work was sponsored by the Space and Missile Defense Command - Battle Lab, under USSTRATCOM Contract FA4600-04D-0008, Delivery Order 0026. demonstrations were designed to evaluate the network features and service capabilities of IRIS. Both types of demonstrations provided complementary insight, operational vs. technical, towards developing a final assessment. The Applied Physics Laboratory of Johns Hopkins University, as the independent Operational Test Agency for the IRIS JCTD, conducted the assessment. The Massachusetts Institute of Technology (MIT) Lincoln Laboratory (LL), the IRIS JCTD Technical Agent, was responsible for developing the customer network emulation, operating the customer network during the tests and collecting and processing the data.

The tests conducted to support the assessment were primarily designed to evaluate the performance of user applications over the IRIS network when subject to various traffic loading conditions, network configurations, and network impairments. The applications used in the assessment were: voice, video teleconference, file transfer, web access and chat. This paper presents some measured results and discusses the technical insights gained through two key demonstrations carried out by the IRIS JCTD. These include the results of a laboratory-based demonstration of an emulated IRIS network with 32 terminals conducted in July 2009; and preliminary results of the final demonstration of the IRIS payload on-board IS-14, carried out during February-March, 2010.

\section{Background}

Transponded ("bent-pipe") satellites have carried Internet traffic for many years, however, satellites with on-board processing (OBP) and regenerative capabilities for broadband commercial communications have only recently become available [3]. Although some of these satellites provide packet-switching functions, an IP router as a communications network node in space did not exist until IRIS.

IP routing in space has the potential to provide significant advantages over other existing systems and could offer important benefits to the end user. These include:

- Instant and seamless IP packet routing across satellite RF channels, transponders, or antenna beams.

- "Point to network" paradigm through a single connection to the satellite router and thereby to any other IRIS terminal.

- "Any-to-any" broadband communications, direct access to the internet and to private networks. 
- "Peer-to-peer" connectivity between user terminals in a single hop, thus eliminating the need for a terrestrial hub.

- Application performance supported by end-to-end Quality of Service (QoS) capabilities and flow control techniques.

- Higher data throughputs from small terminals compared to those on "bent-pipe" satellites, achieved by the higher carrier-to-noise ratio afforded by the on-board modem's signal regeneration capability.

The technical assessment was focused on network functionality and performance in order to validate these claims and assess the utility of IRIS to the end-user. The specific assessment areas consist of: IP routing, QoS, Bandwidth-on-Demand (BoD), network performance and application performance. The following sections describe the IRIS network and the specific features and capabilities available for the lab and field demonstrations and assessment.

\subsection{IRIS Network Architecture}

The IRIS NGGS network consists of the IRIS payload, the IRIS ground terminal nodes and the IRIS NOC. The payload consists of the Programmable Satellite IP Modem (PSIM), the IP Router (IPR) and up and down converters (see Figure 1). The PSIM provides all of the functions of an IP modem ${ }^{2}$ (i.e. encapsulation and segmentation of Layer-3 IP packets, modulation, coding and Layer-2 BoD features). Each 36-MHz IRIS-enabled transponder supports a small set of channels with symbol rates of $1.25,2.5$, and 5 Msps. The IRIS PSIM is connected to three antenna beams of IS-14: C-Band Americas (C-A), Ku-Band Americas (Ku-A), and Ku-Band Europe Africa (Ku-EA).

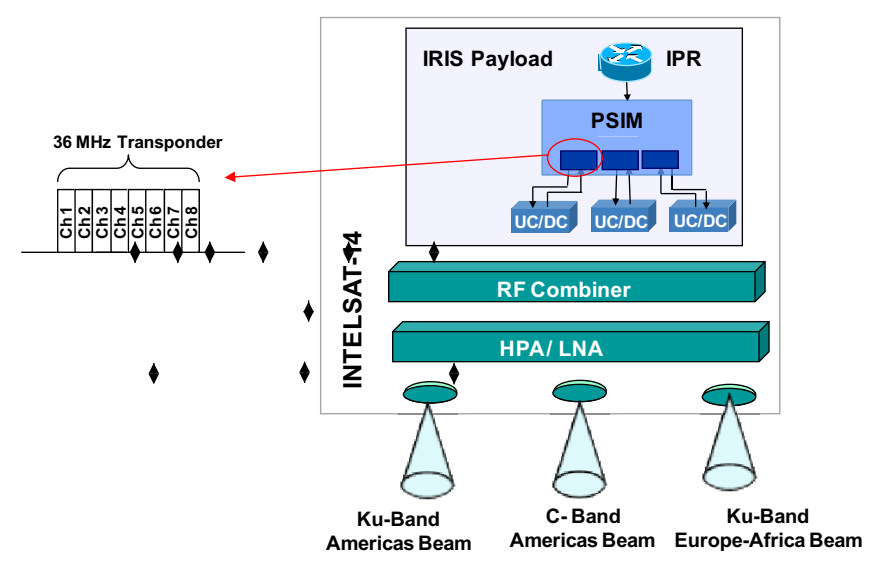

Figure 1: IRIS Payload on INTELSAT-14

Each ground terminal node includes a Linkway modem (LM) and a Cisco Ground Router (CGR). The modems and the PSIM enable dynamic $\mathrm{BoD}$ and support specific Committed

\footnotetext{
${ }^{2}$ The waveform selected for the PSIM is ViaSat's Linkway MultiFrequency TDMA modem.
}

Information Rates (CIR). Each terminal enables a Permanent Virtual Circuit (PVC) with the PSIM. The CGR provides DiffServ-based QoS with traffic conditioning while the LM provides DSCP-based priority Queuing. The CGR interfaces support Dynamic Host Configuration Protocol (DHCP) or External Border Gateway Protocol (eBGP) routing protocols. This allows direct connectivity and network convergence of small and large terminal sites or network nodes. Further details on the IRIS NGGS QoS/BoD architecture can be found in [2]. The IRIS NOC consists of a Network Control Center (NCC) for managing the Linkway modems and the PSIM functions, and a Network Management System (NMS) [4] for managing the ground routers and the IPR.

\subsection{Network Features and Capabilities}

The IRIS Network features and capabilities available for the onorbit demonstration and assessment included:

- Intra-beam, inter-beam and cross-band connectivity.

- BoD capability for dynamic reallocation of bandwidth among all terminals.

- Multiple VPNs through Border Gateway Protocol (BGP) policy-based routing and IPsec encryption.

- VPN isolation to prevent cross-communication between VPNs.

- IP QoS features with Differentiated Services (DiffServ), Weighted Random Early Detection (WRED) and Class Based Weighted Fair Queuing (CBWFQ).

- Connectivity between various types of user networks through eBGP, DHCP and static routing protocols.

- Terminals with multiple service grades ${ }^{3}$ with a choice of access speeds and CIR settings.

- TCP Performance Enhancement Proxy (PEP), compression and caching capability through Cisco's Wide Area Application Services (WAAS) units.

- Public Internet access with global addressing through a dedicated "Internet Gateway"

\section{Network Under Test}

The test bed architectures designed to support the laboratory and field evaluations consisted of the IRIS NGGS network

\footnotetext{
3 According to Cisco's NGGS design, user terminals that choose a "premium service" have a Service Level Agreement (SLA) with a specified CIR while "Best Effort" (BE) users have no service guarantees and no CIR. Terminals with premium service may choose $\mathrm{P} 0, \mathrm{P} 1, \mathrm{P} 2$ or $\mathrm{P} 3$ service grades. $\mathrm{P} 0$ provides the highest data rate and CIR while $\mathrm{P} 3$ provides the lowest data rates and CIR.
} 
(payload, ground terminals, and NOC), and the user network comprised of the government customer emulated network.

\subsection{Lab Emulation Network}

As the JCTD program unfolded, the lab emulation capabilities evolved, becoming increasingly more complex and providing higher fidelity. During the July 2009 lab demonstration, the emulated IRIS network included payload engineering models of the Programmable Satellite IP Modem (PSIM) and the IP Router (IPR). The test bed provided a realistic network scale with a large number of terminals (32 user nodes) that loaded the network, better traffic generation capabilities, and a more refined set of tests and test tools than those used in previous IRIS demonstrations. The laboratory emulation test bed is shown in Figure 2.

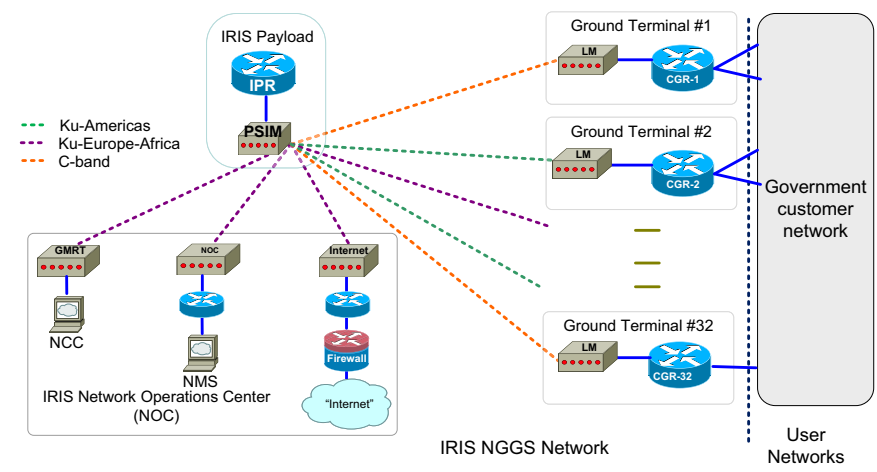

Figure 2: Lab emulation test bed

The PSIM included the three "IRIS-enabled" transponders to emulate "inter-beam," "intra-beam" and "cross-band" connectivity between users. The IRIS portion of the test bed also included three other nodes: the teleport node for Internetlike access; a NOC terminal for NMS functions and a NCC terminal for LM- PSIM performance monitoring and control. The 32 user nodes were assigned to different antenna beams. Each node was configured to represent a different terminal service grade. The hosts at each node were assigned to different closed user groups (VPNs). Some hosts had access to both VPNs and the internet. The "user network" of the test bed was emulated by the Travelling Network and Link Emulation Test $\mathrm{Bed}^{4}$ (TNLET), which emulated the government nodes, and the Lincoln Adaptable Real-time Information Assurance Test bed (LARIAT), that generated the instrumented applications and provided automated management of virtual users.

\subsection{On-Orbit Network}

The test bed for the On-Orbit Assessment (OOA) consisted of two user terminal nodes and the Internet Gateway node. Node-1 was located in Georgia, United States of America while Node-2

\footnotetext{
${ }^{4}$ TNLET and LARIAT were developed by MIT-Lincoln Laboratory.
}

was located in The Hague, Netherlands. INTELSAT's Teleport in Atlanta, Georgia, provided the Internet gateway function and hosted Linkway's NCC. The Cisco RTP node located at Raleigh, North Carolina, provided the IRIS NOC function and enabled a customer Web portal for performance monitoring. Figure 3 illustrates the test bed architecture for the OOA.

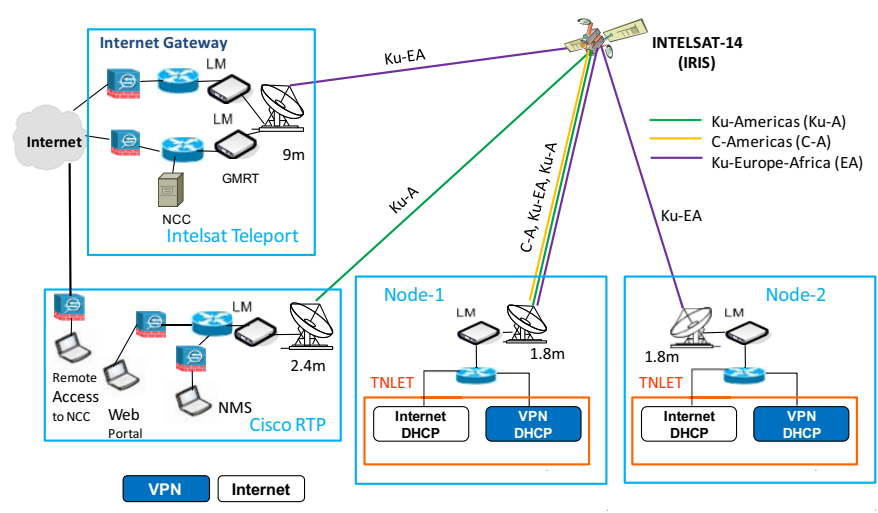

Figure 3: Test Bed Architecture for On-Orbit Assessment

Node-1 had equipment that could operate on either the C-Band Americas (C-A) beam, Ku-Americas (Ku-A) beam, or the $\mathrm{Ku}-$ Europe-Africa (Ku-EA) beam. This node was used to demonstrate cross-band, inter-beam and intra-beam connectivity. Node- 2 was configured to operate only in the $\mathrm{Ku}$ Band Europe-Africa (Ku-EA) beam. The test bed supported two user groups: one "Internet" user group and one VPN user group. The user hosts peered to the CGR through DHCP. All user traffic was emulated by automated instrumented applications.

\subsection{Traffic Generation and Measurement Tools}

The test traffic was generated by TNLET, LARIAT and DVQattest ${ }^{5}$. The instrumented applications consisted of Voiceover IP (VoIP) calls, Video teleconference (VTC), file transfer (FTP) downloading, Web browsing and Instant Messaging (Chat) conversations. TNLET simulated a surrogate Internet, surrogate Intranets, network servers, customer edge devices and end-user workstations. TNLET also generated ping packets between hosts, virtual user's traffic such as FTP file downloads, Internet Web browsing, and Chat conversations at each user node. LARIAT provided automated large-scale management of virtual users and network services such as DNS. DVQattest was used to generate VoIP and Video Tele-Conference (VTC) application traffic at the two user terminals. All sessions were initiated by the emulated virtual user and were marked at their source with appropriate DSCP.

LARIAT and DVQattest were also used to collect performance and traffic statistics for FTP, Web, Chat, VoIP, and VTC.

\footnotetext{
${ }^{5}$ Test tool developed by Telchemy (http://telchemy.com) for generating VoIP and VTC applications traffic.
} 
Netflow was enabled at every CGR in the test bed to collect IP packet data of specific traffic flows. The Linkway NCC provided layer-2 data such as RF burst requests and allocations, per link. Two traffic loading profiles were used. A "Medium traffic" loading profile was used to simulate "normal" traffic conditions at each host and at each terminal. A "Heavy traffic" loading profile was used to simulate periods of intense activity generated by each host of a terminal.

\section{Performance results}

The network and application performance of the IRIS NGGS network was evaluated under various network configurations and traffic loading conditions. This section discusses some results obtained over a series of demonstration activities.

\subsection{Network Performance}

Network performance was assessed through measurements of data throughput collected at various network interfaces, round trip delay (RTD) from host to host and bandwidth allocation to each ground terminal.

\section{Data Throughput}

Figure 4 illustrates data throughput results obtained at Node-2 during the on-orbit tests. Each colour line in the graph represents a different destination IP address of the traffic flowing out of a node. Under heavy loading, the total throughput was about twice than under medium loading. The peak rate was near its maximum capacity (4.23 Mbps).

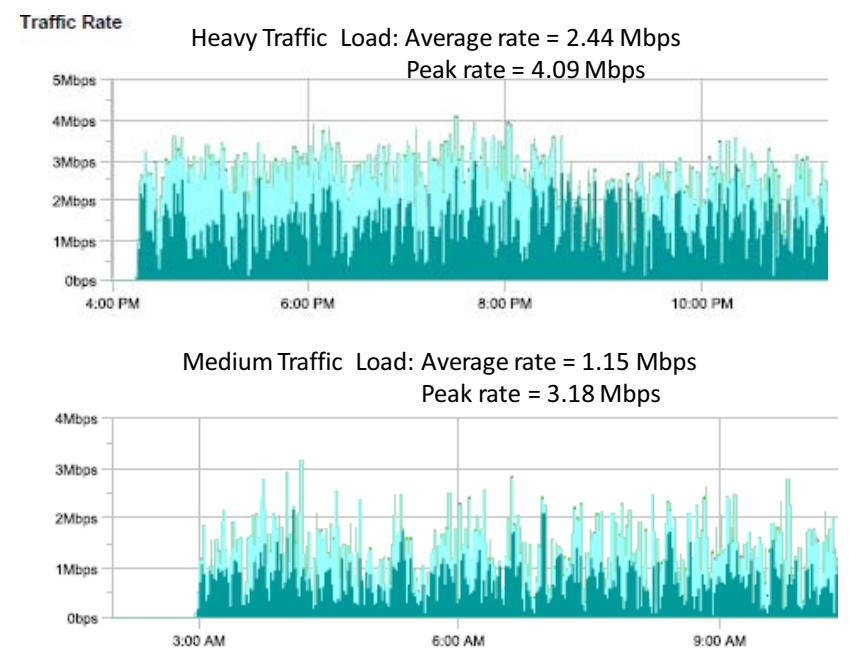

Figure 4: Uplink total throughput of Node-2 under Medium and Heavy traffic loading

\section{Round Trip Delay}

Round Trip Delay (RTD) was measured by sending ping (ICMP) packets marked with one of the four DSCPs (i.e., EF, $\mathrm{AF} 41, \mathrm{AF} 21$, or $\mathrm{BE})$. The results obtained show adequate handling of marked traffic and the QoS solution implemented worked effectively. Table 1 provides a summary of the calculated RTD statistics collected at Node-1 of the OOA under "Medium" and "Heavy" traffic loading conditions. EF-marked packets received the smallest average delay and smallest spread compared to all other classes; BE packets performed the worst and had the largest spread, while the AF41 and AF21 packets were somewhere in between. Furthermore, compared to the medium loading, the results under heavy loading show that the average RTD and distribution spread changed very little for the EF class, increased somewhat for the AF41 and AF21 class and increased significantly for the BE class.

Table 1: RTD Measurements at Node-1

\begin{tabular}{|c|c|c|c|c|c|}
\hline $\begin{array}{c}\text { Traffic } \\
\text { Loading }\end{array}$ & DSCP & $\begin{array}{l}\text { Avg. } \\
\text { (ms) }\end{array}$ & $\begin{array}{c}\text { Std Dev } \\
(\mathrm{ms})\end{array}$ & $\begin{array}{l}\text { Min } \\
(\mathrm{ms})\end{array}$ & $\begin{array}{l}\text { Max } \\
\text { (ms) }\end{array}$ \\
\hline \multirow{4}{*}{ 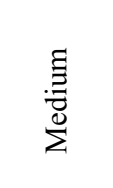 } & $\mathrm{EF}$ & 771.0 & 15.7 & 741.3 & 883.5 \\
\hline & AF41 & 817.0 & 127.2 & 741.7 & 3715.8 \\
\hline & AF21 & 790.4 & 53.8 & 742.2 & 1912.2 \\
\hline & $\mathrm{BE}$ & 902.7 & 282.3 & 741.7 & 3946.8 \\
\hline \multirow{4}{*}{ 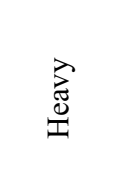 } & $\mathrm{EF}$ & 771.4 & 15.8 & 741.0 & 869.6 \\
\hline & AF41 & 814.5 & 108.6 & 741.9 & 3474.1 \\
\hline & AF21 & 796.8 & 62.9 & 741.5 & 2493.9 \\
\hline & $\mathrm{BE}$ & 957.3 & 339.5 & 743.1 & 3960.9 \\
\hline
\end{tabular}

\section{Bandwidth Resource Allocation}

The bandwidth management function in IRIS is provided by Linkway's NCC. The NCC runs a proprietary Bandwidth on Demand (BoD) algorithm designed to allocate the bandwidth available from each set of channels within a transponder dynamically and with "fairness" among all active terminals that request bandwidth. Terminals with specified CIRs are accommodated first. Then, the NCC allocates any excess bandwidth to all remaining terminals on an as-available basis. During the OOA demonstration, all bandwidth requests were $100 \%$ allocated under both medium and heavy traffic loading. This was due to the small number of terminals (3) and hence the relatively low total traffic volume that did not load the PSIM. However, the results for the lab emulation tests, with a network loaded with 32 ground terminals, are more representative. For example, under light traffic loading P3 service grade terminals got $90 \%$ to $100 \%$ of the requested bandwidth, whereas under heavy loading the resource allocation dropped to $67 \%$. Higher service grade terminals ( $\mathrm{P} 0, \mathrm{P} 1$ and $\mathrm{P} 2)$ got better allocations than $\mathrm{P} 3$ terminals.

\subsection{Applications Performance Under Various Traffic Loading Conditions}

During the OOA the application performance was measured by generating instrumented applications. All application packets 
were marked with appropriate DSCP as defined in the QoS scheme. The class marking agreed was as follows: VoIP (EF), VTC (AF41), FTP (BE), and Web (BE).

\section{VoIP Performance}

The listening and conversation quality of VoIP calls were measured by the "R-factor" and its corresponding Mean Opinion Score (MOS) $)^{6}$. The VoIP application used the ITU defined G.729A voice codec. Table 2 summarizes the performance results of VoIP calls generated by DVQattest under both medium and heavy traffic loading conditions.

Table 2: Average VoIP Performance under Medium and Heavy Traffic Loading

\begin{tabular}{|l|l|l|}
\hline VoIP Performance metrics & Medium & Heavy \\
\hline R-Factor (Listening Quality) & 81.9 & 75.63 \\
\hline R-Factor ( Conversation Quality) & 48.3 & 39.84 \\
\hline MOS (Listening Quality) & 3.90 & 3.63 \\
\hline MOS (Conversation Quality) & 2.37 & 2.08 \\
\hline Packet loss rate & 0.03 & 2.64 \\
\hline Packet discard rate & 0.00 & 0.94 \\
\hline Packet delay variation: PPDV (ms) & 10.23 & 31.53 \\
\hline One-way delay (ms) & 435.55 & 517.77 \\
\hline
\end{tabular}

The results show that the one-way delay (mostly propagation delay) was the major determinant of VoIP performance quality. Furthermore, under heavy traffic loading, VoIP quality was affected by higher packet loss rates, packet discard rates, and PPDV. Upon investigation, it was determined that the unexpected low performance in the heavy traffic case was due to an overloading of the traffic generator rather than the network. The expected results should have shown similar performance for both traffic loading conditions since VoIP packets were marked with the highest EF class. Overall, the VoIP results are considered "fair" (MOS scale) and acceptable to satellite users ${ }^{7}$.

\section{VTC Performance}

VTC performance was measured by the Video Service Multimedia Quality (VSMQ) and Video Service Transmission Quality (VSTQ) [6] scores. ${ }^{8}$ VSMQ considers the effect of picture \& audio quality and audio-video synchronization on

\footnotetext{
${ }^{6}$ The R-factor is measured in a scale 1-100 whereas the MOS is measured in a scale 1-5. ITU Rec. G.107 [5] provides a mapping of RFactor scores to user satisfaction.

7 The ITU [5] has adopted a provisional Advantage Factor of 20 for satellite connections. Hence the measured scores for conversational quality of Table 2 should be increased by $20 \%$.

${ }^{8}$ VSMQ is measured in a scale $0-50$, whereas VSTQ is measured in a scale $0-100$.
}

overall user experience, whereas VSTQ measures the ability of a network to carry reliable video applications. DVQattest generated all VTC applications using the ITU defined H.264 video codec. A theoretical data rate of $156.8 \mathrm{kbps}$ per VTC session was predicted based on frame size and data rate. The VTC performance results shown in Table 3 were calculated for sessions that were successfully set-up during the OOA.

Table 3: Average VTC Performance under Medium and Heavy Traffic Loading

\begin{tabular}{|l|l|l|}
\hline VTC Performance metrics & Medium & Heavy \\
\hline VSMQ & 28.69 & 29.72 \\
\hline VSTQ & 76.56 & 82.11 \\
\hline Packet loss rate & 0.01 & 0.03 \\
\hline Packet discard rate & 0.35 & 0.23 \\
\hline Packet delay variation: PPDV (ms) & 10.34 & 9.31 \\
\hline One-way delay (ms) & 454.56 & 451.75 \\
\hline
\end{tabular}

The results show virtually the same performance under medium and heavy traffic loading. One-way-delay and CODEC-type are the two greatest determinants of VTC performance quality. The QoS implementation provided the appropriate quality for AF41marked traffic and the performance of VTC was acceptable.

\section{Web Performance}

The performance of web page browsing is measured by its session throughput. During the test, the same web page was downloaded multiple times. Since the WAAS units were enabled and included caching, all repeated web page downloads were removed from the calculations to avoid any skewing of the data. Figure 5 shows the average session throughput versus web page size. The results show that Web (marked as BE class) was directly impacted by traffic loading.

- Under heavy traffic loading, the throughput of large web pages (greater than $50 \mathrm{~KB}$ ) was worse than under medium traffic loading. The larger the web page size, the greater the degradation.

- Under both traffic loading conditions, the larger the file size, the greater the session throughput.

- No significant difference in throughput ${ }^{9}$ was observed for the small web pages (less than $50 \mathrm{~KB}$ ) between medium and heavy traffic loading conditions.

\footnotetext{
9 Since FTP runs on top of TCP, the Slow-start algorithm of TCP initially limits data throughput to avoid creating congestion. The TCP connections never reach the maximum window size and hence small data files will generally result in low data throughput in high bandwidth-delay product networks, such as in IRIS.
} 


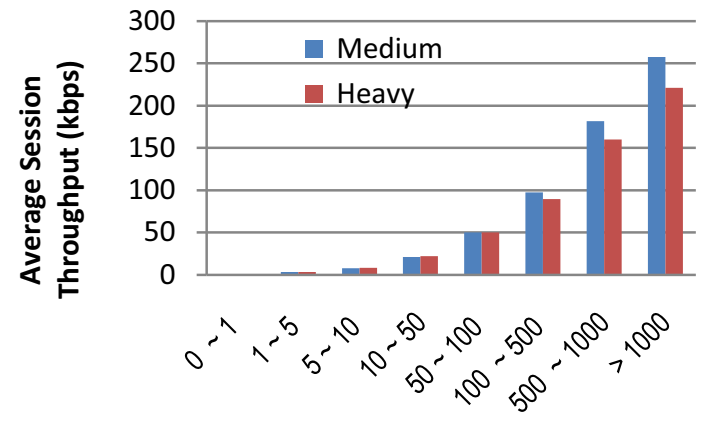

Web Page Size (KB)

Figure 5: Web Performance under Medium and Heavy Traffic

\section{Application Performance with and without PEP}

Two tests were performed to assess the impact stemming from the use of PEP (Cisco's WAAS) in the IRIS network. The first test was run with all three PEP modules enabled; the second test was run with the PEP modules disabled. Both tests were run with a traffic profile containing only FTP traffic consisting of four different file sizes. Figure 7 shows the results of the average session throughput versus the file size of FTP measured with and without PEP.

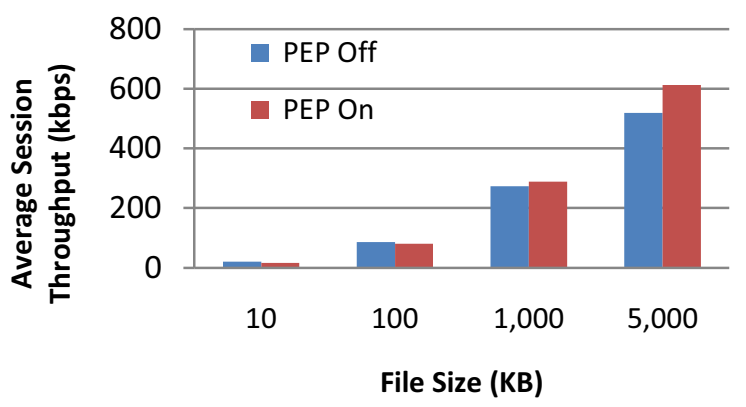

Figure 6: FTP performance with and without PEP devices

We conclude that PEP devices improved the performance of large FTP files $(>100 \mathrm{~KB})$ but that there was little improvement (and even some degradation) for small and medium size files. Through other measurements with random generated FTP files, it was verified that the WAAS compression and caching combined with the PEP functions provided even higher throughputs and thereby reduced even further the consumption of satellite bandwidth.

\section{Assessment of Functionality and Performance}

IRIS provided direct connectivity to different user groups, terminal types and user nodes located in distinct geographical areas. Cross-band, inter-beam and intra-beam connectivity was seamlessly achieved once the user nodes and satellite ground terminals were properly configured. The network supported multiple private enterprise networks, Internet access, and connectivity to the Public Switch Telephone Network (PSTN). The BoD functionality for managing the uplink bandwidth demonstrated the ability to allocate resources dynamically, upon request, to all terminal nodes. The lab emulation tests, which provided the necessary scale to load the network and create congestion conditions, showed that IRIS adequately handled any temporary surges of traffic by rapidly reassigning resources. The end-to-end QoS architecture leveraged differentiated services and effectively demonstrated interoperability of QoS features enabling clear benefits to realtime applications. The quality of VoIP and VTC, which were carried as high priority services, was determined to be acceptable and remained consistent, under various traffic conditions. On the other hand, the performance of nonprioritized packets (FTP, web applications, and chat), running at a best effort level of service varied widely correlating well with network utilization conditions.

\section{Conclusions}

The results presented in this paper and the overall assessment showed that the IRIS network capabilities available for the onorbit demonstration performed adequately, according to the intended design. The quantitative results, together with qualitative observations obtained from the operational demonstrations, which addressed issues such as interoperability, suitability, and operational impact, will be the basis for the final utility assessment of IRIS. The IRIS JCTD Operational Manager will provide the final recommendation on whether the IRIS capability is suitable for the needs of the DOD user.

\section{References}

[1] E. Cuevas, M. Florio, S. Fisher, D. Heuser, G. Jansson, S. Mittal, P. Murray, A. Worthen, S. Yaghmour, "Internet Routing in Space: Prospects and Challenges of the IRIS JCTD”, MILCOM, November 2007.

[2] J.A. Connary, P. Donner, J. Johnson, J. Thompson, "Internet Routing in Space: Architectures for Quality of Service", IEEE Aerospace Conference, March 2009.

[3] E. Cuevas, Z. Tang, "Considerations for the performance evaluation of a geostationary satellite with an on-board internet router", International Communications Satellite Systems Conference, AIAA, June 2009.

[4] J. Johnson, P. Donner, J.A. Connary, J. Thompson, "IRIS NGGS NMS Architecture", IEEEEAC, IEEE Aerospace Conference, March 2009.

[5] International Telecommunication Union, Recommendation ITU-T G.107, "The E-model, a computational model for use in transmission planning," May 2000.

[6] A. Clark, A. Pendleton, "RTCP XR - IP Video Metrics Report Blocks", IETF Internet Draft, December 2006. 